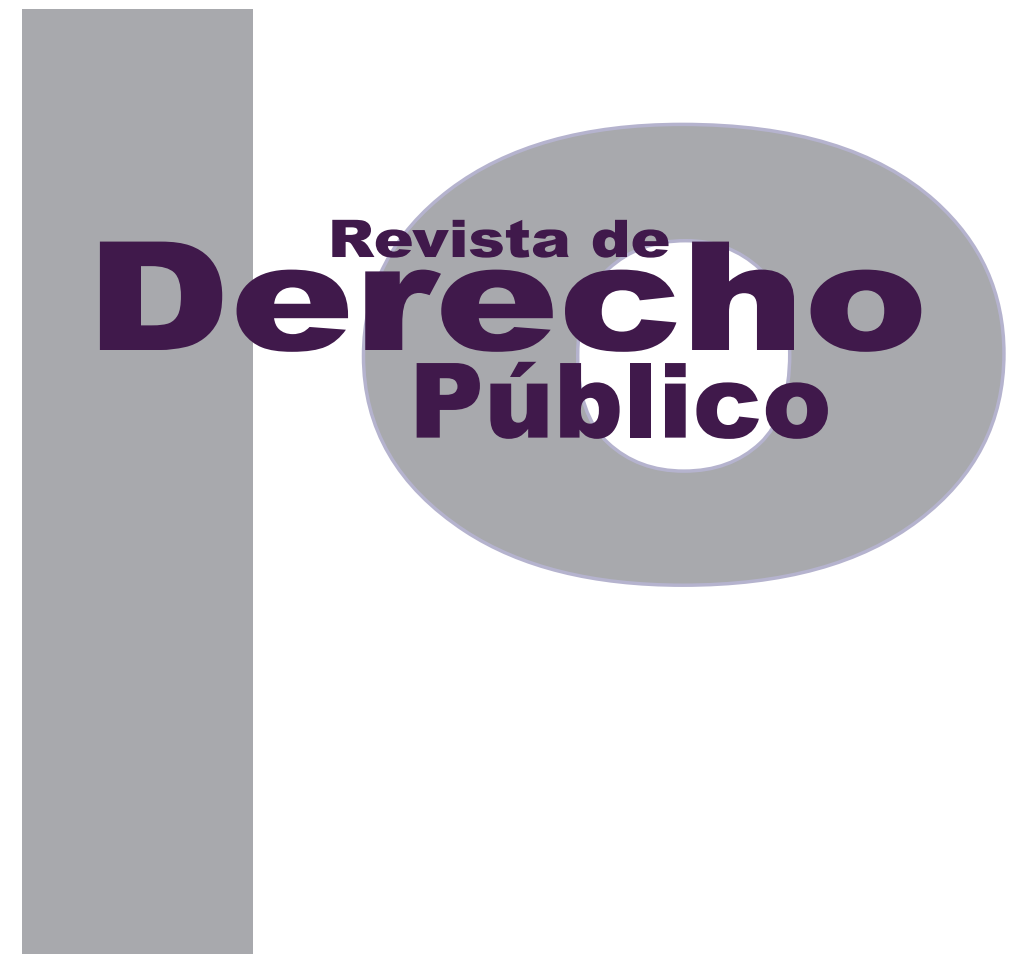

\title{
EL TRASTORNO MENTAL TRANSITORIO CON Y SIN BASE PATOLÓGICA: UNA REVISIÓN DESDE LA MEDICINA LEGAL Y EL DERECHO
}

Carlos Osvaldo de la Espriella Carreño

\author{
Artículo de reflexión
}

Universidad de los Andes

Facultad de Derecho

Revista de Derecho Público N. ${ }^{\circ} 32$

Enero - Junio de 2014. ISSN 1909-7778 


\title{
El trastorno mental transitorio con y sin base patológica: una revisión desde la medicina legal y el derecho
}

\section{Resumen}

El presente artículo ofrece algunas precisiones conceptuales generales acerca del trastorno mental y sus clasificaciones, a saber: trastorno mental permanente, transitorio con base patológica y sin base patológica como causas de inimputabilidad en el régimen jurídico-penal colombiano (CP. Art. 33) y sus consecuencias procesales.

Palabras clave: Trastorno mental, medida de seguridad, inimputabilidad.

\section{The temporary mental disorder with and without pathological basis: A review from the legal medicine and the criminal law}

\begin{abstract}
This article offers certain general conceptual precisions about the mental disorder and its classifications, for instance the permanent mental disorder, the transitory mental disorder with pathological basis and without pathological basis, all causes of imputability absence in the colombian criminal law system (PC. art. 33), and its procedural consequences.

Keywords: Mental disorder, imputability, inimputability, transitory mental disorder, permanent mental disorder, security measures.

\section{O transtorno mental transitório com e sem base patológica: uma revisão desde a medicina legal e o direito}

\section{Resumo}

O presente artigo oferece algumas precisões conceituais gerais sobre o transtorno mental e suas classificações, a saber: transtorno mental permanente, transitório com base patológica e sem base patológica como causas de inimputabilidade no regime jurídico-penal colombiano (CP. Art. 33) e suas consequências processuais.

Palavras-chave: Transtorno mental, medida de segurança, inimputabilidade. 


\section{SUMARIO}

Introducción - I. INIMPUTABILIDAD EN COLOMBIA: ANÁLISIS SUSTANCIAL - A. Trastorno mental - 1. Concepto de trastorno mental - B. Trastorno mental permanente - 1 . Concepto de trastorno mental permanente - 2. Medidas de seguridad por un trastorno mental permanente - $C$. Trastorno mental transitorio - 1. Concepto de trastorno mental transitorio - 2. Consecuencias del trastorno mental transitorio: medidas de seguridad - 3. Subclases de trastorno mental transitorio: a) con base y b) sin base patológica - II. CONCLUSIONES - Bibliografía. 


\title{
El trastorno mental transitorio con y sin base patológica: una revisión desde la medicina legal y el derecho ${ }^{1}$
}

\author{
Carlos Osvaldo de la Espriella Carreño²
}

Introducción

En el presente texto se pretende, por una parte, ofrecer algunas precisiones conceptuales generales acerca del trastorno mental y sus clasificaciones, a saber: trastorno mental permanente, transitorio con base patológica y sin base patológica, como causas de inimputabilidad en el régimen jurídico-penal colombiano (CP. Art. 33), y sus consecuencias procesales; finalmente, se concluirá sobre las consecuencias jurídicas de las clasificaciones realizadas, concretamente en lo referente a las medidas de seguridad procedentes y los distintos fenómenos procesales penales pertinentes.

Cómo citar este artículo: Espriella de la, C. O. (Junio, 2014). El trastorno mental transitorio con y sin base patológica: una revisión desde la medicina legal y el derecho. Revista de Derecho Público, 32.

2 Abogado de la Universidad de los Andes. Candidato a Magister en Derecho procesal de la Universidad Externado. Abogado de la firma Taboada Hoyos abogados. correo: carlos.delaespriella@taboadahoyos.com
Para ello, se analiza tanto la doctrina jurídica internacional como la nacional con respecto a los trastornos mentales en sus diferentes vertientes, así como la literatura médico-legista correspondiente. Además, se estudia la jurisprudencia de la Corte Suprema de Justicia y de la Corte Constitucional colombiana que se ha construido alrededor del tema del presente texto. Finalmente, se precisan las consecuencias jurídicas de cada tipo de trastorno mental y, en particular, las consecuencias de naturaleza procesal que resultan procedentes en cada caso.

\section{INIMPUTABILIDAD EN COLOMBIA: ANÁLISIS SUSTANCIAL}

Para ser imputable se requiere poseer una capacidad psíquica que permita comprender potencialmente el carácter injusto de la acción típica y antijurídica y dirigir las acciones propias conforme a dicha comprensión. Este es el contenido general de la categoría de imputabilidad, que se deriva de la definición negativa prevista 
en el art. 33 de la Constitución Política de 1991, que reza así:

Es inimputable quien en el momento de ejecutar la conducta típica y antijurídica no tuviere la capacidad de comprender su ilicitud o de determinarse de acuerdo con esa comprensión, por inmadurez sicológica, trastorno mental, diversidad sociocultural o estados similares (la expresión subrayada fue declarada exequible por la Corte Constitucional en sentencia C-370 de 2002, M. P.: Eduardo Montealegre Lynett).

El artículo 33 diferencia varios elementos que componen la inimputabilidad, en su orden: el temporal (momento en el que debe darse la situación que causa la inimputabilidad), el normativo, el psicológico (efecto o consecuencia psicológica que se exige para poder pretender la declaratoria de inimputabilidad), y el biológico (causa de la inimputabilidad) (Velázquez, 2009). El presente artículo se enfocará en el elemento biológico únicamente, en concreto, en uno de los elementos biológicos: el trastorno mental.

\section{A. Trastorno mental}

\section{Concepto de trastorno mental}

Con respecto al ingrediente causal o biológico, la norma consagra cuatro posibles causas de la inimputabilidad, siendo la cuarta una cláusula abierta. Así, para el Código Penal (CP) las causas que pueden producir la inimputabilidad son: i) la inmadurez psicológica; ii) el trastorno mental; iii) la diversidad sociocultural; iv) y los estados similares a los anteriores. A continuación se precisará únicamente la causa del trastorno mental, por cuanto ella es el objeto de estudio específico de este artículo.

El trastorno mental, según el art. 33 inc. 1 del $\mathrm{CP}$, es una causa de inimputabilidad, es decir puede dar lugar, si concurren otros requisitos, a que la persona sea declarada inculpable o con culpabilidad disminuida o semiplena por la realización de un injusto en el proceso penal, siempre que se descubran las pruebas de ello en la audiencia de formulación de acusación del proceso penal. ${ }^{3}$ En la mayoría de los casos, la doctrina jurídica considera que quien debe establecer qué es un trastorno mental y de qué clase es el perito médico, con base en el uso de métodos científicos aprobados por la comunidad científica. Esa es la razón por la que su estudio y concepto ha sido desarrollado generalmente por profesionales de la medicina legal.

Los conceptos de trastorno mental se pueden dividir en tres grupos: i) los que definen el trastorno mental por oposición a la normalidad psíquica; ii) los que definen el trastorno mental como una perturbación necesariamente funcional; y iii) los que definen el trastorno mental como una perturbación mental con una consecuencia definida jurídicamente. ${ }^{4}$

3 En efecto, el descubrimiento de las pruebas que se pretenden hacer valer para obtener el reconocimiento de la inimputabilidad, a manera de excepción, debe ser hecho por parte de la defensa, no en la audiencia preparatoria sino en la audiencia de formulación de acusación (art. 344 inc. 2 Ley 906 de 2004).

4 Resulta particularmente interesante la opinión de Juan Oberto Sotomayor sobre el trastorno mental: "La situación de desigualdad de las personas que padecen trastorno mental o inmadurez psicológica (C. P., art. 31) aparece reconocida en forma expresa en el art. 13 de la Const. Pol. Se trata de personas que en razón de su salud mental se encuentran no solo de hecho sino inclusive institucionalmente (C. c. art. 1504) disminuidas de manera considerable en sus posibilidades 
La jurisprudencia de la Corte Suprema de Justicia, al respecto, no ha mantenido una orientación duradera en el tiempo ni ha elaborado reglas de ratio decidendi en las que se opte claramente por alguna de estas posturas. Sin embargo, en los obiter dicta de sus decisiones ha hecho referencias que permiten someramente inferir su posición.

i) El trastorno mental como anormalidad psíquica. El primer grupo de conceptos parte de considerar al trastornado mental como un anormal en un sentido psíquico. Incluso, quienes así piensan opinan que esa "anormalidad" psíquica hace imposible juzgar moralmente -entendiendo ese juicio moral como un juicio de culpabilidad- al autor del injusto penal.

Nereo Rojas (1942, p. 445), por ejemplo toma como punto de partida los planteamientos de Carrara al afirmar que en la enfermedad mental no hay normalidad psíquica, lo que imposibilita una imputación a nivel moral, porque no existe voluntad de ejecutar el hecho. Vargas Alvarado (1983, o. 327) también comparte esta posición, haciendo un especial énfasis en que para estar en presencia de un trastornado mental a éste le debe ser imposible adaptarse socialmente.

La Corte Suprema, en sentencia del 29 de enero de 1999, al referirse a la formulación jurídicopenal colombiana de inimputabilidad, compues-

de participación. En efecto, por un lado la incapacidad de contratación, por ejemplo, implica de por sí una evidente limitación de la vida social para el sujeto, pues restringe sus posibilidades de acceso al mercado, lo cual, en una sociedad de consumo como la nuestra, significa tanto como la desaparición del sujeto como ser social, por lo que de hecho, en el mejor de los casos, se ha visto sometido a una tan paternalista como autoritaria tutela social y estatal" (Sotomayor, 1998, p. 260). ta por causas biológicas principalmente y por consecuencias psicológicas jurídicamente preestablecidas, expresó la siguiente afirmación que deja entrever una concepción del trastorno mental como anormalidad psíquica:

Precisamente, la forma de determinarse a la hora de los acontecimientos (mayormente que antes o después de los mismos) es la expresión más genuina no solo de la normalidad o de una disfuncionalidad psíquica del sujeto, sino también de la capacidad o incapacidad de comprender la entidad del acto que realiza. (Corte Suprema de Justicia, 1999). [Subrayas fuera del texto]. ${ }^{5}$

No compartimos esta primera posición porque la dicotomía normal/anormal no solo no se ajusta a la realidad sino que es inocua. Los parámetros de normalidad siempre resultan arbitrarios e incalculados, y cuando se reconoce que en la realidad la anormalidad es el patrón común, y que existen tendencias de congruencia en grados de anormalidad, el problema pasa a ser de una naturaleza estadística, difícilmente demostrable en el proceso penal, que termina por permitir al juez declarar al procesado anormal y por tanto trastornado mental, con base en su percepción o sus prejuicios.

ii) El trastorno mental como perturbación funcional. El segundo grupo de teorías se refieren al trastorno mental como la perturbación de las funciones biológicas dependientes de la

5 Corte Suprema de Justicia, Sala de Casación Penal, M. P.: Jorge Aníbal Gómez Gallego, Proceso 11058, 29 de enero de 1999. 
psiquis, ${ }^{6}$ trastorno que se identifica como tal si produce una perturbación de las funciones biológicas.

Durán y Carreño (1999, p. 202) identifican a las funciones biológicas como funciones autónomas, y de su existencia depende la presencia de un trastorno mental. Rivera Moreno (2011, p. 190), por su parte, caracteriza aquellas funciones biológicas como fenómenos psíquicos, psicológicos y orgánicos de alteración de la conciencia. ${ }^{7}$ A pesar de que este autor también hace referencia a conceptos como sanidad mental para definir negativamente el trastorno mental, que pudiera considerarse como una reformulación del concepto de normalidad psíquica, se enfoca en las alteraciones de la conciencia, específicamente en la función psicológica y cerebral, por lo que el criterio de diferenciación entre un trastornado y una persona sana deja de ser un criterio de anormalidad.

6 Las funciones biológicas dependiente de la psiquis, o funciones mentales, se dividen en dos grupos: las funciones mentales simples y las funciones mentales superiores. Las funciones mentales simples incluyen las funciones motoras, las sensitivo-motoras, las auditivas y las visuales (perceptivas netamente). Las funciones mentales superiores incluyen, por su parte, la atención (que se divide en atención espontánea y atención voluntaria), la memoria (que tiene tres fases: el aprendizaje, el almacenamiento y el recuerdo, y temporalmente se divide en inmediata, reciente y remota), el lenguaje, el razonamiento abstracto y las actividades gestuales (asociativas especialmente). (Rodríguez Rey, s. f., p. 1 ss.).

7 "La conciencia es el proceso biológico en el cual el individuo mantiene un estado de alerta con pleno conocimiento de sí mismo y de su entorno. Grados de trastorno de la conciencia: Letargia: Consiste en un compromiso incompleto de conocimiento y vigilia. El paciente está desorientado y somnoliento pero se mantiene despierto. Obnubilación: Es un estado de depresión completa de la vigilia, del que el paciente puede ser despertado con estímulos leves. Estupor: Es un estado de depresión completa de la vigilia, del que el paciente puede ser despertado pero solo con estímulos intensos. Los estímulos son generalmente de tipo doloroso (compresión de la raíz ungueal) con una superficie roma. Coma: Constituye la depresión completa de la vigilia de la cual el paciente no puede ser despertado con ningún estímulo." (Hospital infantil de México Federico Gómez, s. f.)
Esta posición también ha sido compartida en diferentes momentos por la jurisprudencia de la Corte Suprema, que, siempre siendo cuidadosa de no utilizar terminología perteneciente a la experticia de la medicina legal, ha identificado la perturbación funcional como un rasgo esencial del trastorno mental:

Lo que la Corte ha expresado sobre el tema, en lo que corresponde al trastorno mental traído a colación por el artículo 210 del Código Penal, es que este estadio se entiende como expresión de inimputabilidad, y, por ello, puede ser de carácter transitorio o permanente (...) en otra oportunidad, refriéndose al mismo tema, reiteró que se tiene entendido que el trastorno mental tiende a identificar estados mas o menos permanentes de enajenación y alteración de las funciones síquicas. (Corte Suprema de Justicia, 2011). [Subrayas fuera del texto]. ${ }^{8}$

8 Corte Suprema de Justicia, Sala de Casación Penal, M. P.: Julio Enrique Socha Salamanca, proceso 34412, 23 de marzo de 2011. En el mismo sentido, Corte Suprema de Justicia, Sala de Casación Penal, M. P.: Sigifredo Espinosa Pérez, proceso 32604, 11 de noviembre de 2009; Corte Suprema de Justicia, Sala de Casación Penal, M. P.: Yesid Ramírez Bastidas, proceso 19747, 3 de diciembre de 2003. Resulta de particular relevancia la definición de trastorno mental que brindó la Corte Suprema de Justicia, Sala de Casación Penal, en sentencia del 13 de octubre de 1982, M. P.: Alfonso Reyes Echandía, y que adoptó la Corte Constitucional en la sentencia C-648 de 2001: "El trastorno mental... es aquella alteración sicosomática que el sujeto sufre en el momento del hecho, de tan profunda intensidad, por tal modo convulsionador de sus esferas intelectiva, volitiva o afectiva que le impide... darse cuenta de la ilicitud de su conducta o determinarse conforme a dicha comprensión. Puede tratarse de una anomalía biosíquica ubicable dentro de la sintomatología clínica propia de una verdadera sicosis, de una grave forma de sicopatía o de una modalidad siconeurítica; pero también es posible que una excepcional y honda aunque pasajera conmoción emotiva que obnubila la conciencia o una también transitoria pero igualmente profunda alteración del intelecto y de la volición, generada por ingestión de bebidas embriagantes o de sustancias narcóticas o estupefacientes causen trastorno semejante." [Subrayas fuera del texto]. Esta definición supone la alteración psicológica de funciones mentales de naturaleza biológica, y de cierta manera constituye una de las pocas definiciones que han sido brindadas por la jurisprudencia colombiana sobre el tema de trastornos mentales en el ámbito penal. 
iii) El trastorno mental como un concepto jurídico. Finalmente, la tercera postura le resta importancia a la definición médica o médico-legista del trastorno mental y, en cambio, considera que todo lo que ocurra en la mente de un sujeto que conlleve a la consecuencia jurídica exigida por el art. 33-1 CP debe ser considerado como trastorno mental.

Cortés y Ortega (2012, p. 308) entienden que el concepto de trastorno mental no es sinónimo de enfermedad mental, y que el primero se caracteriza porque no permite al autor de un hecho comprender o decidir.

La Corte Suprema ha adoptado en reiteradas ocasiones esta posición, ${ }^{9}$ a pesar de que dicha adopción no haya sido resultado de un juicioso análisis conceptual. Un ejemplo de esto se observa en sentencia del 11 de noviembre de 2009, M. P.: Sigifredo Espinosa Pérez: "Con este reconocimiento psiquiátrico se establece plenamente la imputabilidad del procesado, ya que como en el mismo se plasmó, este para el momento de los hechos no padecía trastorno mental alguno, es decir era capaz de autodeterminarse." (Corte Suprema de Justicia, 1999). ${ }^{10}$

Obsérvese cómo la Corte Suprema equipara el concepto de trastorno mental, o más específicamente la ausencia de trastorno mental, a la

9 Esta posición también ha sido asumida por la Corte Suprema, entre otras, en las siguientes sentencias proferidas: Corte Suprema de Justicia, Sala de Casación Penal, M. P.: Mario Mantilla Nougues, 30 de octubre de 1997; Corte Suprema de Justicia, Sala de Casación Penal, Rad. 32604, auto de 11 de noviembre de 2009.

10 Corte Suprema de Justicia, Sala de Casación Penal, M. P.: Sigifredo Espinosa Pérez, proceso 32468, 11 de noviembre de 2009. capacidad de autodeterminación. Como se verá, este trato conceptual indistinto desvirtúa la necesidad de concurrencia de distintos elementos para que proceda la declaratoria de inimputabilidad.

A pesar de mostrar que los conceptos jurídicos y los médicos no son del todo compatibles, no compartimos la tercera posición porque en ella se confunden dos elementos diferentes de la inimputabilidad, a saber: la causa biológica y la consecuencia o el elemento psicológico (es decir, la incapacidad potencial de entender la ilicitud de una conducta o de actuar conforme a ese entendimiento). El efecto psicológico debe ser independiente de la causa biológica, de tal manera que la existencia de un trastorno mental no siempre dé lugar a la identificación de un fenómeno de inimputabilidad. ${ }^{11}$ Consideramos incorrecto también pensar que la mera existencia de una enfermedad ya supone la existencia de un trastorno mental. Es por lo anterior que encontramos correcta la segunda posición y nos adscribimos a ella.

La consideración de que el trastorno mental es la perturbación de las distintas funciones de la psiquis permite, por un lado, no incurrir en la arbitrariedad de definir el trastorno mental como

11 Esta es la razón por la cual tampoco compartimos la posición de la CSJ en una providencia del año 2000: "Ahora bien, el trastorno mental como fuente de inimputabilidad puede ser causado por muy diversos factores: traumáticos, hereditarios, psicológicos, sociológicos y orgánicos; no obstante, en el plano jurídico-penal, como lo ha entendido la Corte, lo que realmente importa no es "el origen mismo de la alteración biosíquica sino su coetaneidad con el hecho realizado, la magnitud del desequilibrio que ocasionó en la conciencia del actor y el nexo causal que permita vincular inequívocamente el trastorno sufrido a la conducta ejecutada." Corte Suprema de Justicia, M. P.: Jorge Aníbal Gómez Gallego, 8 de junio de 2000. 
la "anormalidad en la psiquis", concepto que en la práctica se estructura como una herramienta para demeritar la pluralidad en las diferentes formas de comportamiento e incluso para discriminar otras visiones del mundo $u$ otras formas de actuación amparadas en el derecho al libre desarrollo de la personalidad; por ejemplo, con base en ese concepto se ha considerado a las personas homosexuales como "enfermas". El concepto de trastorno mental como perturbación funcional desliga el contenido de la institución de un ejercicio de normalización de la sociedad, y reduce la arbitrariedad en su uso, pues identificar el trastorno mental con "anormalidad" implica usar un concepto inconstitucional, que termina por ser personalista, homogeneizador y desigual.

Por otro lado, la posición que se adopta en el texto también permite desligar el efecto psicológico, que es un elemento del fenómeno de la inimputabilidad, de la causa de la inimputabilidad denominada como trastorno mental. En realidad, la causa y la consecuencia no pueden ser lo mismo, y menos cuando un enunciado normativo como el art. 33-1 del CP establece unas causas abiertas de inimputabilidad, pero usa en cambio una lista taxativa cerrada para el efecto psicológico requerido. El distanciamiento conceptual de una y otra figura abre la posibilidad de que exista, por ejemplo, un trastorno mental que no derive en una declaratoria de inimputabilidad al no cumplir con el efecto psicológico, como es el caso del sujeto que conduce ebrio y da muerte a un peatón, puesto que a pesar de estar bajo el influjo del alcohol, en tal grado que se le considera bajo una intoxicación aguda, dicha intoxicación no le imposibilita llegar a comprender la ilicitud de su conducta, o comportarse conforme a ese entendimiento.

El concepto de trastorno mental es lo suficientemente amplio como para cobijar toda una serie de perturbaciones funcionales que pueden diferir ampliamente entre ellas, como lo demuestra Fernández Carrasquilla (1998, p. 249) ${ }^{12}$ cuando enumera las posibles alteraciones funcionales que son consideradas como trastornos mentales. El autor se refiere a las enajenaciones mentales como psicosis o demencias, a las psicopatías -incapacidad congénita y constitucional que no permite internalizar normas de las conductas sociales-, la embriaguez, algunos trastornos sexuales, manías, neurosis obsesivas y epilepsias.

Todas las anteriores perturbaciones que constituyen un trastorno mental pueden, a su vez, clasificarse en varios tipos: i) el trastorno mental permanente; ii) el trastorno mental transitorio con base patológica; y iii) el trastorno mental transitorio sin base patológica. ${ }^{13} \mathrm{~A}$ continuación

12 Por lo mismo, Velázquez Velázquez invita a considerar la expresión trastorno mental como un concepto jurídico y no médico: "La expresión trastorno mental tiene un significado jurídico que aleja al jurista de la interminable discusión en torno a la clasificación de las diversas noxas psiquiátricas —aunque no prescinde de la misma-; por eso, no puede interpretarse como si se tratara de un fenómeno médico, sino que ha de otorgársele un contenido amplio, según las necesidades del ordenamiento penal." Velázquez (2009, p. 853).

13 "En cuanto a sus efectos sicosomáticos, el trastorno mental puede ser permanente o transitorio, según que aquellos persistan o tengan precaria duración temporal; el trastorno mental transitorio, a su vez, puede subsistir cuando el juez deba decidir sobre la situación jurídica definitiva del procesado, o haber desaparecido ya, en cuyo caso se habla de un trastorno mental transitorio sin secuelas." Reyes Echandía (1994, p. 197). 
se precisan las diferencias entre unas y otras categorías.

\section{B. Trastorno mental permanente}

\section{Concepto de trastorno mental permanente}

El trastorno mental permanente es aquella perturbación funcional psíquica que persiste en el tiempo de manera continua. La alteración mental permanente constituye el efecto de la perturbación funcional psíquica, por lo que tanto la causa, esto es la perturbación funcional psíquica, como la consecuencia, esto es la alteración mental, deben perdurar temporalmente.

Algunos de los trastornos mentales permanentes más importantes son aquellos que hacen parte del género de las psicosis, como son las esquizofrenias en sus diferentes vertientes, a saber: la esquizofrenia tipo paranoide, la esquizofrenia tipo desorganizado, la esquizofrenia tipo catatónico, la esquizofrenia tipo indiferenciado y la esquizofrenia tipo residual. Cada uno de los anteriores trastornos tiene una sintomatología particular (ver DMS-IV-TR), ${ }^{14}$ pero su característica común es que generan una pérdida de contacto con el mundo real por medio de alteraciones en la percepción, denominadas delirios.

La característica de perturbación limitada en el tiempo es la que casi todos los autores subra-

14 Se refiere al Manual diagnóstico y estadístico de los trastornos mentales. (IV ed.). Clasificación de los trastornos mentales, que proporciona descripciones claras de las categorías diagnósticas de la Asociación Americana de Psiquiatría (APA). yan. Por ejemplo, Rojas (1942, pp. 435-436) señala que esa persistencia excluye de esta categoría fenómenos como el de la ebriedad y el sonambulismo.

Otros autores han considerado que la alienación mental debe ser incurable, por oposición al trastorno mental transitorio. Se cree también que, por oposición al trastorno mental transitorio, el permanente debe depender de una causa intrínseca, esto es, que nace y se desarrolla en el propio sujeto que la padece. Sin embargo, conceptuamos que las anteriores características no son esenciales y exclusivas del trastorno mental transitorio, toda vez que estas, como se verá, pueden ser compartidas con las otras categorías de trastornos mentales, sin necesidad de desvirtuarlas y entenderlas como trastornos mentales permanentes.

\section{Medidas de seguridad por un trastorno mental permanente}

Las medidas de seguridad que proceden para una persona que ha actuado bajo un trastorno mental permanente se regulan en el art. $70 \mathrm{del}$ CP. ${ }^{15}$ La regulación establece la internación del

15 Art. 70 del CP: "Al inimputable por trastorno mental permanente, se le impondrá medida de internación en establecimiento psiquiátrico, clínica o institución adecuada de carácter oficial o privado, en donde se le prestará la atención especializada que requiera. Esta medida tendrá un máximo de duración de veinte (20) años y el mínimo aplicable dependerá de las necesidades de tratamiento en cada caso concreto. Cuando se establezca que la persona se encuentra mentalmente rehabilitada cesará la medida. Habrá lugar a la suspensión condicional de la medida cuando se establezca que la persona se encuentra en condiciones de adaptarse al medio social en donde se desenvolverá su vida. Igualmente procederá la suspensión cuando la persona sea susceptible de ser tratada ambulatoriamente. En ningún caso el término señalado para el cumplimiento de la medida podrá exceder el máximo fijado para la pena privativa de la libertad del respectivo delito." 
sujeto penalmente condenado en un establecimiento especializado en donde se le preste la atención que necesite para su curación, tutela o rehabilitación (CP, art. 5). Se impone un límite temporal de veinte años de internación como máximo, si el máximo de la pena para ese delito excede ese límite, o el máximo de la pena si ella es menor a veinte años, y como mínimo, en ese marco penal, se establece el tiempo necesario requerido por el condenado para lograr su rehabilitación mental. Sin embargo, el término real de la internación depende de las necesidades del tratamiento en cada caso, y por tanto la medida de seguridad debe finalizar cuando ya no se requiera, o cuando se cumpla con el máximo posible de tiempo de internación para cada caso, así el sujeto no haya logrado su curación. Es importante resaltar que las medidas no pueden ser indefinidas, basadas en la peligrosidad del sujeto, como ocurría en el CP de $1936 .^{16}$

Por otro lado, la suspensión de la medida de seguridad procede en dos circunstancias: i) cuando se considera por dictamen médico que la persona está en condiciones mentales que le permitan adaptarse al medio social donde vivirá; o ii) cuando el tratamiento necesitado pueda ser ambulatorio. Para que se suspenda la medida de seguridad se requiere que un informe pericial previo determine la presencia de alguna de las dos circunstancias por las que procede la

16 "En efecto, la influencia del positivismo criminológico italiano fue evidente en el Código Penal de 1936, que consagró algunos de sus principios fundamentales, tales como el de responsabilidad legal y peligrosidad. (...) Esta posición cambia con la expedición del C.P. de 1980. (...) Este viraje hacia el derecho penal de culpabilidad y del acto en la legislación colombiana, vino acompañado de la adscripción de nuestro C.P. a una determinada concepción de la culpabilidad y por ende de la imputabilidad" (Sotomayor, 1996, p. 204). suspensión (CP, art. 79). Así mismo, la suspensión de la medida de seguridad se puede revocar, exigiéndose para ello, también, un informe pericial previo que considere necesario el cumplimiento de la medida de seguridad original (CP, art. 78), basado en consideraciones médicas, o que se evidencie el incumplimiento de las obligaciones fijadas en la diligencia de compromiso (Código de Procedimiento penal, art. 469, en adelante C. de P. P.). El juez competente para conocer de la solicitud de la suspensión de la medida de seguridad, así como de su revocatoria, es el juez de ejecución de penas y medidas de seguridad, o de la primera instancia, según el caso. Todo lo anterior es aplicable para los inimputables por trastorno mental transitorio con base patológica.

En caso de que se suspenda la medida de seguridad, o esta cese porque se cumplieron los compromisos procesales, se podrá imponer como medida accesoria la libertad vigilada del inimputable, que implica el cumplimiento de las siguientes obligaciones: i) residir en determinado lugar por un término no mayor de tres años; ii) no concurrir a determinados lugares hasta por un término de tres años; y iii) presentarse periódicamente ante las autoridades encargadas de su control hasta por tres (3) años (CP, art. 74$).{ }^{17}$

17 La posibilidad de solicitar la libertad vigilada del inimputable no depende ni se afecta por el tiempo de internación preventiva (a manera de medida cautelar) que haya sucedido dentro del proceso, como lo ha establecido la jurisprudencia, incluso basada en otras codificaciones anteriores: "Significa lo anterior que el inimputable por trastorno mental permanente, se hará acreedor en el curso del proceso al beneficio de "libertad vigilada", cualquiera sea el término cumplido en internación preventiva, cuando el psiquiatra forense determine que su afección psíquica ha alcanzado cierto grado de evolución satisfactoria que le permita reintegrarse a la sociedad sin representar un peligro para sí mismo o para los demás. 
Para que pueda efectuarse la suspensión de la medida de seguridad o su sustitución a la libertad vigilada, sin embargo, es necesario prestar una caución, personalmente o por medio de un representante legal.

Necesariamente, para los trastornos mentales permanentes y para los trastornos mentales transitorios con base patológica, cuando se reconozca el fenómeno de la inimputabilidad al sujeto debido a esto, solamente puede proceder uno de los tipos de medidas de seguridad contemplados en el CP, art. 69, que sería la internación en establecimiento psiquiátrico o clínica adecuada. Ello se deriva del CP, art. 70 incs. 3 y 4, que establece de manera expresa la necesidad de la imposición de esa medida de seguridad, en particular para el caso de los trastornos mentales permanentes, y del CP, art. 71 incs. 3 y 4 , que establece lo mismo para los trastornos mentales transitorios con base patológica. A pesar de ello, el juez de ejecución de penas y medidas de seguridad está facultado para modificar la sanción según lo estime necesario y adecuado, de acuerdo con los informes de los equipos terapéuticos responsables del cuidado, tratamiento y rehabilitación del sujeto inimputable condenado (C. de P. P., art. 38 num. 1 y num. 6 inc. 2).

Hay que anotar que la no declaratoria de inimputabilidad, cuando haya lugar, permite que pro-

Esta concesión podrá ser revocada por el funcionario de conocimiento, de oficio o a solicitud de parte, cuando el procesado incumpla las obligaciones adquiridas o cuando el perito médico oficial aconseje la continuación de la medida de internación (art. 427, inciso final del C. de P. P.)." (Corte Suprema de Justicia, Sala de Casación Penal, M. P.: Fernando E. Arboleda Ripoll, proceso 10612, 11 de julio de 1996). ceda el recurso de casación por violación directa o indirecta de la ley sustancial, según el caso.

La medida de seguridad debe ser impuesta, en principio, por el juez de conocimiento en la audiencia de lectura del fallo (C. de P. P., arts. 40 y 452) y en la sentencia condenatoria, mientras que el juez de ejecución de penas y medidas de seguridad ordena la internación, comunicando dicho acto al sistema general de seguridad social en salud, quien asignará el centro de rehabilitación correspondiente, que será puesto a disposición por el Instituto Nacional Penitenciario y Carcelario, INPEC (C. de P. P., art. 466).

\section{Trastorno mental transitorio}

Es común encontrar en las providencias de la Corte Suprema de Justicia, casi como única referencia al trastorno mental transitorio, las conclusiones a las que llegan en cada proceso los peritos, sobre su existencia en contextos en donde un injusto penal fue cometido bajo un estado de consumo de alcohol o sustancias psicoactivas. ${ }^{18}$

Sin embargo, la Corte Suprema no ha ofrecido una caracterización juiciosa de dicho trastorno, por considerar que este es un concepto de índole netamente médico-legista, en el que la experticia médica es la que decide qué es y qué no es un trastorno mental transitorio. Por lo anterior, a continuación se analiza el contenido de dicha categoría.

\footnotetext{
18 Entre este tipo de providencias se encuentran las siguientes de la Corte Suprema de Justicia, Sala de Casación Penal, procesos 34412, 23 de marzo de 2011; 3543518 de abril de 2012 y 3803914 de marzo de 2012.
} 


\section{Concepto de trastorno mental transitorio}

i) Definición. La definición de trastorno mental transitorio que consideramos acertada es la siguiente: una perturbación en las funciones dependientes de la psiquis del sujeto, que produce una alteración de duración breve en la capacidad cognitiva y volitiva, y que se debe a una causa externa o interna con respecto al sujeto que la padece.

En lo que atañe a los elementos que deben concurrir para que un trastorno mental se pueda clasificar como transitorio, la doctrina postula varios elementos, de los cuales solo algunos deben ser reconocidos como esenciales.

ii) Requisitos exigidos por los diversos autores

1- El primer requisito es que la alteración tenga una duración breve (Gisbert Calabuig, 1994; ${ }^{19}$ Ángulo González, 201020). La doctrina que se

19 Gisbert Calabuig (1994) define estos requisitos: "Características: 1. Desencadenado por una causa inmediata y de fácil demostración. 2. Aparición brusca o, por lo menos, rápida. 3. Duración breve. 4. Curación rápida, completa, sin secuelas y sin probabilidades de repetición. 5. Base patológica probada. 6. Anulación completa del libre albedrío e inconsciencia u obnubilación temporal. (...) Base patológica. Debe existir una predisposición. Esto explica la razón por la cual no todas las personas, ante los estímulos desencadenantes, reaccionan con un trastorno mental transitorio. (...) Causas. Sobre esta predisposición van a actuar los estímulos desencadenantes, que son agentes o influjos externos o exógenos. Estos agentes pueden ser físicos y psíquicos. Entre los físicos, hay infecciones, intoxicaciones y traumatismos. Los psíquicos están representados por las llamadas reacciones psicógenas. Estas no son más que reacciones anómalas mentales debidas a estímulos vivenciales de origen psíquico. Su aceptación como causas de trastorno mental transitorio será tanto mayor conforme más se aparten de las reacciones habituales del individuo normal."

20 "el trastorno mental transitorio es una perturbación mental que anula las facultades psíquicas con privación de la voluntad y el raciocinio, de duración limitada, que desaparece sin dejar secuelas. Los trastornos mentales transitorios son estados de la perturbación mental pasajeros y curables, debido a causas ostensibles sobre una base patológica refiere al tema del trastorno mental transitorio concuerda en que la esencia de este radica en la brevedad de la alteración funcional, esto es, la transitoriedad de la perturbación funcional psíquica. Reyes Echandía (1994, p. 197) afirma que la desaparición de la alteración en un breve tiempo puede deberse tanto a un tratamiento clínico como a una desaparición espontánea.

2- Un segundo requisito del trastorno mental transitorio consiste en la curación total sin secuelas, según opinión, entre otros, de Vargas Alvarado (1983) y Gisbert Calabuig (1994, p. 752) quien considera que exige la curación total de la patología, pues su existencia depende de la intervención de un agente externo que, al no presentarse, permite que el sujeto esté psíquicamente equilibrado.

En todo caso, existe un problema normativo. E Código penal colombiano contempla la existencia de una clase de trastorno mental transitorio con base patológica. ${ }^{21}$ Si se acepta la curación total y sin secuelas como requisito del trastorno mental transitorio, querría decir que la base patológica, en los casos de trastorno mental

probada, cuya intensidad puede llegar a producir trastorno en la comprensión y voluntad.(...) Los elementos que componen el trastorno mental transitorio son: 1) origen: se trata de reacciones a agentes venidos de fuera, por motivos físicos, psíquicos o mixtos. Los trastornos mentales transitorios no vuelven a repetirse si no vuelve a darse el motivo externo. 2) comienzo: a de ser producido por una causa externa inmediata, necesaria y fácilmente evidenciable. Es decir, que debe existir una secuencia entre la causa y el efecto. (...) 3) duración: tiene terminación con curación completa, recuperando su estado anterior al episodio de la perturbación mental. 4) base patológica: suele observarse la existencia de un terreno predisponente, pero no es indispensable." (Angulo González, 2010, pp. 442-484).

21 Artículo 75 CP. TRASTORNO MENTAL TRANSITORIO SIN BASE PATOLÓgICA. Si la inimputabilidad proviene exclusivamente de trastorno mental transitorio sin base patológica no habrá lugar a la imposición de medidas de seguridad. 
transitorio con base patológica, tendría que dejar de existir, pues si permanece no habría una ausencia completa de secuelas o una curación absoluta. Es decir, que aquellos trastornos que se fundamentan en una base patológica incurable pero que no implican una alteración funcional constante, no podrían ser considerados trastornos mentales transitorios porque su base patológica constituirá una secuela permanente, pero tampoco podrían ser trastornos mentales permanentes porque la duración de la alteración derivada del trastorno no es de este tipo, lo que dejaría estos especiales eventos en un limbo jurídico inaceptable.

Por la misma razón, cuando la doctrina propone que para la existencia de un trastorno mental transitorio sea necesaria la existencia de una base patológica (Vargas Alvarado, 1983; Gisbert Calabuig, 1994), esta interpretación no puede ser conciliada con la legislación colombiana. En efecto, el art. 75 del CP contempla la posibilidad de la existencia de un trastorno mental transitorio sin base patológica, por lo que sería una contradicción conceptual establecer como requisito de los trastornos mentales transitorios la existencia de una base patológica. En fin, el requisito de curación total sin secuelas y el de la existencia de una base patológica no son aplicables a los trastornos mentales según el ordenamiento jurídico-penal colombiano.

3- Un tercer requisito es la causa inmediata (Vargas Alvarado, 1983; Angulo González, 2010). Se refiere a que la causa de la alteración funcional psíquica se deba a una causa temporalmente inmediata. Se establece, de esta manera, un nexo causal entre una causa y el efecto de la inimputabilidad que, para el efecto, es la alteración funcional del sujeto.

Un ejemplo de ello es cuando se está frente a un síndrome psicótico - un estado de alucinación- y se alega que este se debió a que el individuo hace un mes consumió alucinógenos, como los hongos con psilobicina, pero dicho sujeto padece de esquizofrenia. En este caso no cuenta la causa inmediata -el ácido-, sino la causa crónica -la esquizofrenia que se viene presentando desde hace un tiempo-que no es categorizable como trastorno mental transitorio sino como un trastorno mental permanente.

4- El cuarto requisito es la causa externa (Angulo González, 2010; Gisbert Calabuig, 1994). Parte de la doctrina especializada sostiene que la alteración psíquica debe ser causada por un agente externo al sujeto. Por el contario, autores como Vargas Alvarado no consideran necesario este elemento para que se dé un trastorno mental transitorio. Nuestra posición al respecto es que, en el caso del trastorno mental transitorio con base patológica, la causa externa no es un requisito, o puede concurrir con una causa interna, mientras que en el caso del trastorno mental transitorio sin base patológica sí es necesaria la causa externa como determinante de la alteración psíquica. ${ }^{22}$

\footnotetext{
22 La causa interna se refiere al origen de la alteración psíquica producida en un trastorno mental transitorio con base patológica, en donde dicho origen reside en la individualidad del sujeto, de manera biológica, $y$, aunque su residencia en el ser del individuo alterado no exige una permanencia perenne, sí es un origen que, salvo intervención psicológica o psiquiátrica, o incluso farmacológica, goza de una vocación de indemnidad (Gisbert Calabuig, 1994).
} 
También se discute si la no existencia de posibilidades de repetición es un requisito del trastorno mental transitorio (Vargas Alvarado, 1983; Gisbert Calabuig, 1994). Este elemento se considera derivado de la exigencia de una causa externa. Se dice que en el trastorno mental transitorio, mientras la causa externa no vuelva a desatarse, es seguro que no aparecerá nuevamente la alteración funcional. Sin embargo, consideramos que este no debe ser un requisito del trastorno mental transitorio, o debe entenderse de una manera diferente, porque las posibilidades de repetición de la causación pueden darse incluso en un trastorno mental transitorio, cuando exista una base patológica determinada que no sea curable. Esta posición parte de la idea de que la ausencia de secuelas como requisito del trastorno mental transitorio no debería existir, por cuanto aquel es uno de los factores que diferencian un trastorno mental transitorio sin base patológica de un trastorno mental transitorio con base patológica.

5- El último requisito sería la aparición brusca de la alteración psíquica (Vargas Alvarado, 1983, p. 330). Según este autor, si la aparición no es brusca, al menos debe ser rápida (cercana a la causa). No se observa cuál es la necesidad, no obstante, de este requisito, salvo reiterar la existencia de un nexo de causalidad entre la causa de la alteración y la alteración psíquica.

Este requisito, en particular, fue utilizado por la Corte Suprema, en sentencia del 23 de marzo de 2011, para caracterizar el trastorno mental transitorio sin base patológica:
Pero además, cuando lo que determina la inimputabilidad es el acaecimiento de un trastorno mental transitorio no preordenado, esto es, debido a la aparición súbita e involuntaria de una situación patológica o no, que impide obrar con capacidad de comprensión y autodeterminación respecto del hecho criminoso, y luego desaparece sin dejar secuelas, el agente no obra en ninguna de las formas de culpabilidad, de suerte que al ser excluida esa categoría no puede imponerse pena y menos una medida de seguridad ante la inexigibilidad de la conducta adecuada a la norma. ${ }^{23}$ [Subrayas fuera del texto].

En conclusión, consideramos que los requisitos de los trastornos mentales transitorios son los siguientes: i) la duración breve de la alteración y ii) una causa inmediata. El resto, a saber: i) la curación total y rápida sin secuelas; ii) la causa externa; y finalmente iii) la no existencia de posibilidades de repetición, son factores que permiten distinguir entre un trastorno mental transitorio con base patológica y un trastorno mental transitorio sin base patológica y, más específicamente, determinar qué debe entenderse por base patológica.

\section{Consecuencias del trastorno mental transitorio: medidas de seguridad}

Las medidas de seguridad que proceden para una persona que padece un trastorno mental transitorio se regulan en el art. $71^{24} \mathrm{y}$ en el art.

23 Corte Suprema de Justicia, Sala de Casación Penal, M. P.: Julio Enrique Socha Salamanca, proceso 34412, 23 de marzo de 2011.

24 "Al inimputable por trastorno mental transitorio con base patológica, se le impondrá la medida de internación en establecimiento psiquiátrico, 
$75^{25}$ del CP. La única medida de seguridad aplicable para estos trastornos es la internación del sujeto penalmente condenado, en un establecimiento especializado en donde se le preste la atención que necesite para su curación, tutela o rehabilitación (CP, art. 5). El límite temporal de la medida de seguridad es de diez años de internación como máximo - si el máximo de la pena para ese delito excede ese límite, o el máximo de la pena si ella es menor a diez años-y como mínimo el tiempo necesario requerido por el condenado para lograr su rehabilitación mental. El tiempo de duración concreto de la internación depende de las necesidades del tratamiento en cada caso; por tanto, la medida de seguridad debe finalizar cuando ya no se requiera, o cuando se cumpla con el máximo posible de tiempo de internación así el sujeto no haya logrado su curación.

Para el trastorno mental transitorio con base patológica, la suspensión de la medida de seguridad procede en las mismas dos circunstancias que para los inimputables por trastorno mental

clínica o institución adecuada de carácter oficial o privado, en donde se le prestará la atención especializada que requiera. (...) Esta medida tendrá una duración máxima de diez (10) años y un mínimo que dependerá de las necesidades de tratamiento en cada caso concreto. La medida cesará cuando se establezca la rehabilitación mental del sentenciado. (...) Habrá lugar a la suspensión condicional de la medida cuando se establezca que la persona se encuentra en condiciones de adaptarse al medio social en donde se desenvolverá su vida. (...) Igualmente procederá la suspensión cuando la persona sea susceptible de ser tratada ambulatoriamente. (...) En ningún caso el término señalado para el cumplimiento de la medida podrá exceder el máximo fijado para la pena privativa de la libertad del respectivo delito."

"Si la inimputabilidad proviene exclusivamente de trastorno mental transitorio sin base patológica no habrá lugar a la imposición de medidas de seguridad. (...) Igual medida procederá en el evento del trastorno mental transitorio con base patológica cuando esta desaparezca antes de proferirse la sentencia. (...) En los casos anteriores, antes de pronunciarse la sentencia, el funcionario judicial podrá terminar el procedimiento si las víctimas del delito son indemnizadas." permanente: i) cuando se considera por dictamen médico que la persona está en condiciones mentales que le permitan adaptarse al medio social donde vivirá; o ii) cuando el tratamiento necesitado pueda ser ambulatorio. Para que se suspenda la medida de seguridad se requiere que un informe pericial previo determine la presencia de alguna de las dos circunstancias por las que procede la suspensión (CP, art. 79). Así mismo, la suspensión de la medida de seguridad se puede revocar, siempre que medie un informe pericial previo que considere necesario el cumplimiento de la medida de seguridad original (CP, art. 78), basado en consideraciones médicas, o que se evidencie el incumplimiento de las obligaciones fijadas en la diligencia de compromiso (C. de P. P., art. 469). El juez competente para conocer de la solicitud de la suspensión de la medida de seguridad, así como de su revocatoria, es el juez de ejecución de penas y medidas de seguridad, o de primera instancia, según el caso.

Cuando se suspenda la medida de seguridad, o esta cese porque se cumplieron los compromisos procesales, se podrá imponer la libertad vigilada del inimputable como medida accesoria, lo que implica el cumplimiento de las siguientes obligaciones: i) residir en determinado lugar por un término no mayor de tres años; ii) no concurrir a determinados lugares hasta por un término de tres años; y iii) presentarse periódicamente ante las autoridades encargadas de su control hasta por tres (3) años (CP, art. 74).

Para que pueda efectuarse la suspensión de la medida de seguridad o su sustitución a la liber- 
tad vigilada, sin embargo, es necesario prestar una caución, personalmente o por medio de un representante legal.

De la misma manera que para los trastornos mentales permanentes, para los trastornos mentales transitorios con base patológica, cuando se reconozca el fenómeno de la inimputabilidad al sujeto debido a esto, solamente puede proceder uno de los tipos de medidas de seguridad contemplados en el $\mathrm{CP}$, art. 69, que sería la internación en establecimiento psiquiátrico o clínica adecuada. Ello se deriva del CP, art. 71 inc. 3 y 4, que establece de manera expresa la necesidad de la imposición de esa medida de seguridad, en particular para el caso de los trastornos mentales transitorios con base patológica. A pesar de ello, el juez de ejecución de penas y medidas de seguridad está facultado para modificar la sanción según lo estime necesario y adecuado, de acuerdo con los informes de los equipos terapéuticos responsables del cuidado, tratamiento y rehabilitación del sujeto inimputable condenado (C. de P. P. art. 38 num. 1 y num. 6 inc. 2).

La medida de seguridad debe ser impuesta, en principio, por el juez de conocimiento en la audiencia de lectura del fallo (C. de P. P. art. 40 y 452) y en la sentencia condenatoria, mientras que el juez de ejecución de penas y medidas de seguridad ordena la internación, comunicando dicho acto al sistema general de seguridad social en salud, quien asignará el centro de rehabilitación correspondiente, que será puesto a disposición por el Instituto Nacional Penitenciario y Carcelario (C. de P. P., art. 466).
Finalmente, si la base patológica del trastorno mental transitorio con base patológica desaparece antes de proferirse la sentencia, no hay lugar a la imposición de una medida de seguridad (art. 75 inc. 2, CP).

En el trastorno mental transitorio sin base patológica no hay lugar a la imposición de una medida de seguridad. ${ }^{26}$

\section{Subclases de trastorno mental transito- rio: a) con base y b) sin base patológica ${ }^{27}$}

Es absolutamente escaso el desarrollo teórico sobre la distinción entre el trastorno mental transitorio con base patológica y aquel sin base patológica tanto en la literatura médico-legal como en la literatura científica y en la jurídicopenal. Todavía es más desconcertante que los

26 Esta consecuencia procesal para la inimputabilidad derivada de trastornos mentales transitorios sin base patológica encuentra antecedentes en la regulación de trastornos mentales transitorios sin secuelas que regía en el Código penal de 1980, como lo evidencia la aclaración de voto del magistrado Fernando E. Arboleda Ripoll, en la sentencia de la Corte Suprema de Justicia del 14 de noviembre de 2002, proceso 15856: "Afirmar que el caso se regula por el artículo 33 del Código de 1980 en razón a que el procesado para el momento de realización de los hechos padecía trastorno mental sin secuelas; declarar que por corresponder la situación al supuesto normativo invocado no hay lugar a imponer medida de seguridad alguna, y no obstante esa conclusión asumir el tribunal que está autorizado para someter a la persona a tratamiento psiquiátrico, determinarlo en su tipo y duración, y así ordenarlo, ¿no es acaso exceder el marco de posibilidades establecido por la norma? ¿y ello no es interpretación errónea?” (Corte Suprema de Justicia, Sala de Casación Penal, M. P.: Jorge Córdoba Poveda, proceso 15856, 14 de noviembre de 2002).

27 "el trastorno mental puede ser transitorio, si cesa en un periodo de tiempo más o menos corto, el cual puede ser, a su vez, con base patológica o sin base patológica. La jurisprudencia española ha definido el trastorno mental transitorio como "todo aquel de causa inmediata, necesaria y fácilmente evidenciable, de aparición más o menos brusca, de duración, en general, no muy extensa y que termina por la curación sin dejar huella, producido por el choque de un agente exterior, cualquiera sea su naturaleza: es decir, una verdadera reacción de situación que produce en el individuo la alteración de su mente, en términos tales que le hace irresponsable de los actos en aquél momento ejecutados por él mismo". Agudelo Betancur (2011, p. 377). 
tratadistas de una y otra disciplina consideren que el desarrollo (teórico y conceptual) de estas figuras debe ser realizado por los estudiosos de la disciplina contraria. Para los médicos, la distinción entre la existencia e inexistencia de una base patológica no es un concepto preciso desde la perspectiva médica, porque puede tener variadas connotaciones; por lo que la base patológica es explicada a partir de la óptica normativa o jurídica. De la misma manera, los juristas consideran que la conceptualización sobre qué debe entenderse por base patológica se debe dar a partir de la ciencia médica y no por el derecho penal, pues la ciencia y sus clasificaciones son cambiantes, aunque deban adaptarse a los criterios de existencia o ausencia de una base patológica.

Esto ha llevado a que la distinción aludida no sea desarrollada por ninguna de las dos disciplinas de manera suficiente, salvo por una escasa doctrina que ha intentado profundizar al respecto; situación de abandono que ha sido puesta en evidencia por Gaviria Trespalacios (2005), quien identifica el problema conceptual incluso con la legislación que contemplaba en vez de trastornos mentales con y sin base patológica, los trastornos mentales con y sin secuelas.

Por lo anterior, y como objetivo principal de este texto, pretendemos ofrecer un concepto sobre la institución trastorno mental con base patológica y sin base patológica.

Las doctrinas médico-legales y jurídico-penales han hecho ciertas distinciones entre estas categorías. Por una parte, se ha dicho que los trastornos mentales transitorios con base patológica se ubican o se presentan sobre una personalidad psicopática, anormal o morbosa ${ }^{28}$ utilizando los tres anteriores términos como sinónimos, mientras que en los trastornos mentales transitorios sin base patológica se dan situaciones mentales anormales pero no morbosas. ${ }^{29}$ Fernández Carrasquilla (1998, p. 251) es uno de los autores que utiliza el concepto de morbosidad para diferenciar entre un trastorno mental permanente y uno transitorio, sin ofrecer mayor precisión conceptual.

Sin embargo, no hay claridad sobre qué quiere decir una "personalidad morbosa" o una "alteración morbosa". El Diccionario de la lengua española define morboso como sinónimo de enfermo, y como causa o consecuencia de una enfermedad. ${ }^{30}$ Según esta definición, en caso de existir un trastorno que sea diagnosticado como una enfermedad existirá una base patológica. La dificultad con esta opción radica, por una parte, en que el trastorno mental, para haber sido considerado como tal, debía ser categorizado como una enfermedad o patología y, por la otra parte, que en la ciencia médica existe una discusión

28 Sin utilizar aquellos términos, pero fundamentalmente dirigiéndose a la misma idea, Gaitán Mahecha (1999, p. 181) se refiere al tópico: "4 Es indiferente [según la legislación de su época] que el trastorno sea producido por un estado patológico -episódico desde luego- o que sea determinado por causas meramente psíquicas como la sugestión. $5^{\circ}$ No debe ser buscado de propósito por el agente, porque en tal caso constituiría actio libera in causa".

29 Según el Diccionario de la lengua española morboso es un adjetivo y significa "enfermo, que causa enfermedad, o concierne a ella", o "que provoca reacciones mentales moralmente insanas o que es resultado de ellas" (Real Academia Española, 2013).

30 Real Academia Española (2013). 
constante entre lo que debe entenderse por patología o lo que no debe entenderse como tal. ${ }^{31}$

Esta dificultad, entonces, hace que exista una inseguridad jurídica absoluta, y que todos los trastornos mentales transitorios deban ser considerados con base patológica, precisamente porque son trastornos mentales. En efecto, para que un fenómeno sea un trastorno mental es necesaria una correlación entre los efectos y una determinada o posible patología, ya que las alteraciones funcionales psíquicas se agrupan mediante patologías por la Clasificación Internacional de Enfermedades (CIE, 10 revisión) y por el Manual diagnóstico y estadístico de trastornos mentales (DSM IV). Si se determina que debe existir una alteración funcional psíquica para que se esté frente a un trastorno mental transitorio con base patológica, todos los trastornos mentales con las características de transitorio tendrían que ser clasificados como transitorios con base patológica, algo que no coincide con nuestro sistema legal.

2- Un segundo criterio de diferenciación sería la existencia de una causa externa como factor desencadenante de la alteración. Esta posición considera que la causa externa no es un requisito material para la existencia de un trastorno mental transitorio, sino que es un criterio que diferencia entre el trastorno mental transitorio con y sin base patológica, con lo que estamos

31 En el presente momento se discute por parte de la Asociación de Psiquiatría Americana si las Ilamadas adicciones psicológicas, como por ejemplo la adicción a los videojuegos, deben ser consideradas como patologías o no. La polémica se desarrolla a raíz de la edición de la quinta versión del Manual diagnóstico y estadístico de trastornos mentales. de acuerdo. Esta posición es presentada por Ferré, Núñez y Ramírez (2010, p. 445), quienes afirman que un trastorno mental transitorio sin base patológica se caracteriza porque su causa es exógena al sujeto trastornado.

Aunque el criterio de la causa externa puede servir para distinguir entre la existencia o no de la base patológica, no es suficiente porque, como toda aseveración de una causalidad natural diferenciable y única, implica ficciones de simplificación de la realidad. Por esto, y a pesar de que existiese una causa externa que pudiera a simple vista considerarse como la conditio sine qua non de la alteración, podría haber otras causas que la produjeran. Es el caso del adicto a las sustancias, que si bien su alteración o intoxicación se produce por cuenta del consumo de sustancias, sin su trastorno de dependencia a la misma la alteración psicológica no se hubiese producido. Este ejemplo muestra entonces por qué la causa externa no es un requisito, ya que se trata de un trastorno mental transitorio con base patológica a pesar de la concurrencia entre una causa interna y una externa.

Bajo esta posición, el adicto no podría acceder a un centro de rehabilitación proveído por el Estado bajo la forma de una medida de seguridad, sino que necesariamente quedaría indemne de dicha medida pues se considera que, dado que existió una causa externa como lo es la ingesta de sustancias, el trastorno consecuente debe clasificarse como trastorno mental transitorio sin base patológica, y la ausencia de la base patológica procesalmente implica la no imposición de una medida de seguridad. Usando este criterio como 
proponen los autores, la única manera que posibilitaría el acceso de un adicto reconocido como inimputable a un centro de rehabilitación, sería el caso en el que la alteración se debió a un síndrome de abstinencia o a un delirium tremens, trastornos que, como se verá, no requieren del uso de sustancias para su desencadenamiento.

3- Un tercer criterio planteado por la literatura es el de la "lesión". Desde esta perspectiva, el trastorno mental transitorio con base patológica implica una lesión a nivel anatómico, que es transitoria y, por consiguiente, curable, a diferencia de aquellas lesiones permanentes identificables como trastornos mentales permanentes. El trastorno mental sin base patológica, en cambio, sería una alteración que no presenta lesiones ni cambios anatómicos. ${ }^{32}$ Esta postura teórica es defendida por Uribe y Uribe (1981, p. 1021), quienes diferencian entre sicopatías por lesión, que pueden ser orgánicas (con lesión duradera pero no necesariamente definitiva) o sicopatías toxi-infecciosas (con lesiones transitorias y curables), y las sicopatías y sicosis constitucionales, que no presentan alteraciones biológicas significativas.

Este planteamiento se puede criticar porque, aún en un evento ampliamente reconocido como un trastorno mental transitorio sin base patológica, como lo es la embriaguez común que logra el efecto psicológico de la inimputabilidad-si se demuestra médicamente la afecta-

32 Se considera que un buen criterio para determinar la existencia o no de los cambios anatómicos es la muerte de algún elemento orgánico, ya sea a nivel micro, como por ejemplo la muerte celular, como también la muerte de un tejido celular o elemento orgánico más complejo. (Uribe Cualla y Uribe González, 1981). ción neuronal o hepática del cuerpo y por tanto que el trastorno cause la muerte de células o neuronas, la embriaguez sería constitutiva de trastorno mental transitorio con base patológica, por lo que se llegaría al absurdo de tener que aplicar una medida de seguridad en un centro de rehabilitación para un procesado, que, evidentemente, no lo necesita. A pesar de ello, se puede considerar que el criterio de la lesión, y por tanto de la existencia de secuelas constitutivas de lesión, puede ser un factor que, combinado con el criterio de la causa externa, provea elementos suficientes para determinar la existencia de una base patológica o la inexistencia de esta. Ello permite que la CSJ considere que, por ejemplo, las secuelas son relevantes para la determinación de la aplicación o no de una medida de seguridad (CSJ, proceso n. ${ }^{\circ} 34412$, del 23 de marzo de 2011, p. 19).

4- Finalmente, el criterio de la probabilidad de repetición de la alteración psíquica es ofrecido por autores como Vargas Alvarado y Gisbert Calabuig, para determinar la presencia de una base patológica. En sentido político criminal, su aplicación provee a los operadores médicolegistas y jurídicos de una herramienta para prevenir que un sujeto pueda volver a presentar una alteración de esta índole. En el caso del sujeto que padece de un síndrome de dependencia, por ejemplo, se puede determinar que tiene una alta probabilidad de volver a experimentar la alteración cuando luego de un consumo habitual y abusivo de sustancias estas se suspendan súbitamente. En este caso, lo político-criminalmente adecuado será internar al inimputable con el fin de prevenir una muy posible nueva al- 
teración, que con probabilidad se traduzca en un acto delictivo interno (reincidente).

A pesar de que el criterio de la probabilidad de repetición se funda en la imposición de una sanción basada en un análisis de proclividad del sujeto al delito, y bajo este entendimiento se pretendería alcanzar una declaración de responsabilidad penal semiplena con base en un juicio exclusivo sobre la personalidad del autor o cómplice, este se ajusta al fin de las medidas de aseguramiento y no de la pena, en particular bajo la causal de peligrosidad para la comunidad e incluso para la víctima (C. de P. P., art. 308). Y es que la proclividad no se refiere a la criminalidad, sino a la alteración que ponga en peligro a las víctimas o a la comunidad. Suponiendo que el Estado debe utilizar las medidas de aseguramiento como herramientas de protección sobre la comunidad y las víctimas, usar la probabilidad de repetición de la alteración como criterio para determinar, en últimas, si procede o no una medida de aseguramiento, constituye un seguimiento por demás cercano a las pretensiones del legislador al establecer cuáles debían ser los fines de dichas medidas.

Por ello, se concluye que para lograr determinar de una manera adecuada la existencia o inexistencia de una base patológica se debe recurrir como criterios informativos, en primer lugar, a la causa externa como elemento esencial de la ausencia de base patológica; en segundo lugar, a la existencia o inexistencia de lesiones anatómicas, o secuelas; y, en tercer lugar, a las probabilidades de repetición de la alteración, fundadas en criterios de la experiencia. Lo anterior, teniendo en cuenta, lógicamente, los diagnósticos clínicos realizados por los expertos de la medicina legal.

\section{CONCLUSIONES}

Desafortunadamente, el contenido de la categoría de trastorno mental no ha sido desarrollado con suficiencia en el ámbito jurídico-penal colombiano, tampoco por los expertos en medicina legal ni por los profesionales del derecho, estos últimos independientemente de su vinculación a la judicatura o a otros espectros de la práctica jurídico-penal. Esto ha hecho que exista una indeterminación conceptual, que genera una situación de inseguridad jurídica al interior del sistema penal colombiano, y eventualmente se presta para consecuencias jurídicas desiguales para casos iguales.

Para ofrecer una solución a dicha indeterminación, por medio de una propuesta conceptual diferenciamos el trastorno mental transitorio con base patológica del trastorno mental transitorio sin base patológica. Para ello, proponemos que los requisitos para que un trastorno mental sea considerado transitorio sin base patológica han de ser los siguientes: i) que exista una causa externa como elemento desencadenante; ii) que unas lesiones anatómicas, denominadas coloquialmente "secuelas", no se hallen presentes con anterioridad al hecho; y iii) que sean reducidas las probabilidades de repetición de la alteración. Así mismo, concluimos que la diferencia entre un trastorno mental permanente y un trastorno mental transitorio radica en que el primero 
es una perturbación funcional psíquica que se caracteriza por mantener en el tiempo la alteración de las funciones, mientras que el trastorno mental transitorio se caracteriza por una duración breve de la alteración y una causa inmediatamente anterior a la alteración en el tiempo.

Esta conceptualización es el resultado de la desestimación de diversas posiciones doctrinales, especialmente, sobre los elementos que constituyen las categorías estudiadas. Esta desestimación la realizamos principalmente por medio del estudio de la sistematización de dichos elementos dentro de un espectro jurídico específico como lo es el sistema penal colombiano.

De la misma manera, identificamos la consecuencia procesal de una y otra clasificación. Para los trastornos clasificados como transitorios sin base patológica demostramos cómo el ordenamiento jurídico-penal establece que frente a estos, en caso de una declaratoria de inimputabilidad, no sea posible imponer ni una pena ni una medida de seguridad. Respecto a los trastornos mentales con base patológica, advertimos cómo la normativa jurídico-penal establece que cuando el juez declare la inimputabilidad en la sentencia condenatoria solo sea posible imponer una medida de seguridad consistente en la internación en un establecimiento adecuado, según el caso, con una duración máxima hasta de diez años o hasta el máximo de la pena privativa de la libertad para el delito sancionado, y sin un mínimo de duración. También, para este tipo de trastornos mentales transitorios existe la posibilidad de suspender la medida de seguridad cuando se observe que el tratamiento puede continuar ambulatoriamente, o cuando el sujeto internado haya obtenido las capacidades suficientes para vivir en la sociedad.

\section{Referencias}

Agudelo Betancur, N. (2011). Elementos de la culpabilidad. En Lecciones de derecho penal, parte general. ( $2^{\mathrm{a}}$ ed.). Bogotá: Universidad Externado de Colombia.

Agudelo Betancur, N. (1994). La inimputabilidad penal. ( $2^{\circ}$ ed.). Bogotá: Librería La Constitución Ltda.

Agudelo Betancur, N. (1993). Trastorno mental transitorio como causal de inimputabilidad penal. Bogotá: Linotipia Bolívar.

Angulo González, R. D. (2010). Medicina forense y criminalística: Sistema penal acusatorio. ( $3^{\mathrm{a}}$ ed.). Bogotá: Editorial Doctrina y Ley Ltda.

Colombia. Ley 906 de 2004. Código de Procedimiento penal.

Colombia. Ley 599 de 2000. Código Penal.

Colombia. Código Nacional de Tránsito.

Colombia. Constitución Política de 1991.

Corte Constitucional. Sentencia C-370 de 2002.

Corte Suprema de Justicia. Sala de Casación Penal, proceso n. 10612 de 11 de julio de 1996. 
Corte Suprema de Justicia. Sala de Casación Penal, proceso n. 32468 de 30 de octubre de 1997.

Corte Suprema de Justicia, proceso n. ${ }^{\circ} 11058$ de 29 de enero de 1999.

Corte Suprema de Justicia, proceso n. ${ }^{\circ} 15856$ de 14 de noviembre de 2002.

Corte Suprema de Justicia, proceso n. ${ }^{\circ} 19747$ de 3 de diciembre de 2003.

Corte Suprema de Justicia. Sala de Casación Penal, proceso n. ${ }^{\circ} 20637$ de 12 de octubre de 2006.

Corte Suprema de Justicia. Sala de Casación Penal, proceso n. ${ }^{\circ} 32604$, auto del 11 de noviembre de 2009.

Corte Suprema de Justicia. Sala de Casación Penal, proceso n. 34412 de 23 de marzo de 2011.

Corte Suprema de Justicia. Sala de Casación Penal, proceso n. ${ }^{\circ} 35435$ de 18 de abril de 2012.

Corte Suprema de Justicia. Sala de Casación Penal, proceso n. ${ }^{\circ} 38039$ de 14 de marzo de 2012.

Cortés Caballero, C., y Ortega Moreno, H. (2012). Juristas y medicina: tratado de medicina legal. ( $4^{\mathrm{a}}$ ed.). Bogotá: Ediciones Doctrina y Ley Ltda.
Durán Robles, L., y Carreño Salazár, M. I. (1999). Principios de psiquiatría forense. ( $3^{\mathrm{a}}$ ed.). Medellín: Señal Editorial.

Estrada Vélez, F. (1981). Derecho penal. Parte general. ( $2^{\mathrm{a}}$ ed.). Bogotá: Librería del Profesional.

Fernández Carrasquilla, J. (1998). Derecho penal fundamental. Tomo II. Bogotá: Editorial Temis.

Ferré Olivé, J. C., Nuñez, M. A., y Ramírez Barbosa, A. (2010). Derecho penal general colombiano. Parte general. Bogotá: Ibañez.

Gaitán Mahecha, B. (1999). Curso de derecho penal general. Bogotá: Ediciones Lerner.

Gaviria Trespalacios, J. (1982). Enfoque psiquiátrico del trastorno mental como fuente de inimputabilidad. Revista Colombiana de Psiquiatría, 1, 47-62.

Gaviria Trespalacios, J. (Diciembre, 2005). La inimputabilidad: concepto y alcance en el código penal colombiano. Revista Colombiana de Psiquiatría, 34(1), 26-48.

Gisbert Calabuig, J. A. (1996). Psiquiatría legal y forense, I. Madrid: Editorial Colex.

Reyes Echandía, A. (1994). Derecho penal, parte general. (11 ${ }^{a}$ ed.). Bogotá: Editorial Temis.

Reyes Echandía, A. (1984). La Inimputabilidad. Bogotá: Universidad Externado de Colombia. 
Rivera Moreno, Á. (2011). Manual práctico de medicina legal para abogados. Cúcuta: Universidad Libre de Colombia.

Rojas, N. Medicina Legal. ( $5^{\mathrm{a}}$ ed.). Buenos Aires: Librería El Ateneo Editorial.

Sotomayor A., J. O. (1998). Inimputabilidad y sistema penal. Bogotá: Temis.

Uribe Cualla, G., Uribe González, C. (1981). Medicina legal, toxicología y siquiatría forense. (11 ${ }^{\mathrm{a}}$ ed.). Bogotá: Editorial Temis.
Vargas Alvarado, E. (1983). Medicina legal: compendio de ciencias forenses para médicos y abogados. ( $3^{a}$ ed.). Costa Rica: Lehman Editores.

Velásquez, F. V. (2009). Derecho penal, parte general. Medellín: Librería Jurídica Comlibros.

Velázquez Velázquez, F. (2004). Manual de Derecho Penal, Parte General. (2ª ed.) Bogotá: Temis. 